\title{
Neonatal and Pediatric Respiratory Care: What Does the Future Hold?
}

\author{
Robert M DiBlasi RRT-NPS FAARC and Ira M Cheifetz MD FAARC \\ Respiratory Research: Why Is It So Difficult? \\ Sharing Data Between Children and Adults \\ Noninvasive Ventilation of Premature Infants: Do We Really Need to \\ Intubate? \\ Neonatal Invasive Mechanical Ventilation \\ Advances in the Management of Pulmonary Hypertension \\ Inhaled Medical Gases: More to Breathe Than Oxygen \\ Resuscitation in the Delivery Room: Lung Protection From the First Breath \\ Surfactant: Neonates and Beyond \\ Asthma 2015 and Beyond \\ Aerosol Delivery: An Old Topic With New Data \\ Airway Maintenance and Clearance: How to Stay Out of Trouble \\ ECMO: Moving at the Speed of Light \\ Disaster Planning for Pediatrics \\ Conclusions
}

\begin{abstract}
Neonatal and pediatric respiratory care continues to move forward at a truly impressive pace. Recent technologic advances and an increasing number of randomized clinical trials are leading to improved outcomes for neonates, infants, children, and adolescents with respiratory illness. The goals of this 47th ReSPIRATORy CaRe Journal Conference were to review pertinent recent advances in neonatal and pediatric respiratory care and, more importantly, to offer thoughts and perspectives for the future of our field. It is important to note that of the prior 46 Journal Conferences, only 2 have been dedicated to neonatal and/or pediatric respiratory care topics. It is our hope that the publication of these proceedings will provide respiratory therapists, physicians, and other members of the clinical care team a foundation on which to ponder the future of neonatal and pediatric respiratory care. Key words: neonatal; pediatric; respiratory care; mechanical ventilation; acute lung injury; respiratory failure. [Respir Care 2011;56(9):1466-1480. (C) 2011 Daedalus Enterprises]
\end{abstract}

\section{Respiratory Research: Why Is It So Difficult?}

Martha Curley, from the Children's Hospital of Philadelphia, began with a provocative discussion of the diffi-

Mr DiBlasi is affiliated with the Center for Developmental Therapeutics, Seattle Children's Research Institute, and with the Department of Respiratory Care, Seattle Children's Hospital and Research Institute, Seattle, Washington. Dr Cheifetz is affiliated with the Division of Pediatric Critical Care Medicine, the Pediatric Intensive Care Unit, Pediatric Respiratory Care, and the Extracorporeal Membrane Oxygenation Program, Duke Children's Hospital, Durham, North Carolina.

Mr DiBlasi and Dr Cheifetz presented a version of this paper at the 47th Respiratory Care Journal Conference, "Neonatal and Pediatric Respiratory Care: What Does the Future Hold?" held November 5-7, 2010, in Scottsdale, Arizona. culties often encountered in clinical trials within the respiratory care field. ${ }^{1}$ The key question addressed was, why are pediatric data so limited? The potential answers included:

\footnotetext{
Mr DiBlasi has disclosed relationships with Monaghan Medical and GE Healthcare. Dr Cheifetz has disclosed relationships with PhilipsRespironics, Covidien, Discovery Laboratories, and Teleflex.

Correspondence: Robert M DiBlasi RRT-NPS, Seattle Children's Research Institute - Respiratory Care, Center for Developmental Therapeutics, 1900 Ninth Avenue, Seattle WA 98101. E-mail: robert.diblasi@ seattlechildrens.org.
}

DOI: $10.4187 /$ respcare. 01505 
- An insufficient number of mechanically ventilated children, especially those with substantial lung injury, to support large-scale randomized controlled trials (RCTs)

- Heterogeneity of the population to be studied in terms of age, size, and medical and surgical diagnoses

- Variable expression of respiratory disease, especially across the age range seen in pediatrics

- Loss of equipoise secondary to the extrapolation of data from previous neonatal and/or adult clinical trials (eg, low $\mathrm{V}_{\mathrm{T}}$ ventilation for adult acute lung injury [ALI])

So what have we done over the years without definitive pediatric data? Often, clinicians have simply learned to function without definitive evidence for their management approach. Pediatric clinicians have become comfortable (for right or for wrong) extrapolating data from the neonatal and adult worlds. But Curley stressed the clear need to do better in the future. A key recommendation was to build a strong program of research rather than performing isolated studies. This approach allows for the formation of a solid foundation so that future studies can grow from prior successes. Investigator-initiated RCTs are optimally conducted as the capstone project within a program of research. ${ }^{2}$

It must be stressed that building a program of research takes time, persistence, and stable and adequate funding. An excellent example of this approach is the pediatric prone positioning study. In 1999, Curley and others systematically reviewed prone positioning of patients with acute respiratory distress syndrome (ARDS). ${ }^{3}$ In 2000, Curley et al conducted a single-center pilot study of early and repeated prone positioning in pediatric patients with ALI. ${ }^{4}$ And in 2005, Curley et al reported a multicenter study of prone positioning in children with ALI. ${ }^{5}$

Another key point for the success of RCTs includes the use of established networks to efficiently and effectively share resources and to draw from a larger population base. Examples of such established networks include the Pediatric Acute Lung Injury and Sepsis Investigators (PALISI) Network, ${ }^{5-9}$ the Collaborative Pediatric Critical Care Research Network(CPCCRN), ${ }^{10-13}$ and the Australian and New Zealand Intensive Care Society (ANZICS) Clinical Trials Group. ${ }^{14-16}$ Among such networks, as well as in general, Curley reminded us to choose our collaborators wisely, based on credibility, cooperation, availability, and a track record of success. This important advice is applicable both to senior investigators when choosing potential sites for a multicenter study as well as to junior investigators when choosing mentors.

In concluding her presentation, Curley provided the general advice to "think differently about clinical research." Specifically, clinical researchers should utilize multidisciplinary teams and thus leverage the strengths of individual team members, and collaborate with teams that have a strong record of success. Furthermore, a paradigm shift is needed in the approach to clinical studies, with an increased focus on qualitative methods in the early phases of a program, an emphasis on building on prior successes (ie, research teams and programs should not disband after a success), and a greater reliance on teleresearch, especially in the design phase for larger prospective trials.

One should consider unpacking the syndromes. Rather than studying ARDS as an entity, study components of the syndrome (eg, fluid or sedation management). For success, it may be necessary to refine clinically important outcomes. Specifically, outcomes must be important enough to convince clinicians to change their clinical practice in response to a positive study. It should be noted that using mortality as a primary outcome variable, in addition to the ethical issues it raises, is not practical in pediatric ALI studies, as it is likely to be impossible to recruit a sufficient number of subjects to demonstrate an effect. ${ }^{17}$

\section{Sharing Data Between Children and Adults}

In follow-up to Curley's presentation, Ira Cheifetz discussed the void of definitive data in the area of pediatric ALI and reviewed the areas in which pediatric clinicians may be able effectively to extrapolate from the available data on adult ALI. ${ }^{18}$ This presentation built on Curley's explanations for the void of pediatric data by reviewing a key publication from the PALISI Network. ${ }^{6}$ In that pediatric weaning study, over 6,400 infants and children were screened. However, only 1,096 (17.1\%) required mechanical ventilation for greater than 24 hours, and 303 (4.7\%) were enrolled. Cheifetz echoed some of Curley's remarks by noting that the void of pediatric data is related to a combination of a limited patient population, relatively short duration of mechanical ventilation for the vast majority of infants and children, substantial clinical variability among patients, management differences between ICUs, and often a lack of equipoise.

Cheifetz's presentation focused on several of the large ARDS Network studies, starting with low-tidal-volume $\left(\right.$ low- $\mathrm{V}_{\mathrm{T}}$ ) ventilation. Although definitive data exist in the adult world that a $6 \mathrm{~mL} / \mathrm{kg} \mathrm{V}_{\mathrm{T}}$ benefits patients with ALI, as compared to a $12 \mathrm{~mL} / \mathrm{kg} \mathrm{V}_{\mathrm{T}},{ }^{19}$ no similar data exist for the management of infants and children. As the basis for this entire discussion, the clinical adage that children are not simply small adults must be acknowledged. But this acknowledgment must go further: infants are not simply small adolescents. When deciding whether adult data can be safely and accurately extrapolated to pediatrics, we must ask critical questions about the population studied, the effect size, the disease processes, the individual patient characteristics, and, more generally, the population in ques- 
tion. A large child or adolescent would clearly mimic the physiology of an adult more closely than would an infant.

Thus, the pediatric clinician is left without clear guidance in determining the optimal $\mathrm{V}_{\mathrm{T}}$ for a child with ALI. It would seem reasonable to extrapolate from the available adult data and use a $6 \mathrm{~mL} / \mathrm{kg} \mathrm{V}_{\mathrm{T}}$. In support of this approach is the lack of any better data, the similarity of ARDS in patients beyond infancy, the lack of any known adverse effects of this approach (albeit based on adult data and pediatric clinical experience, but not pediatric data), and basic physiologic principles. However, many questions remain. Would a $V_{T}$ of less than $6 \mathrm{~mL} / \mathrm{kg}$ lead to even better results (as may be true for adult patients as well)? Where should the delivered $\mathrm{V}_{\mathrm{T}}$ be measured (ie, at the ventilator or with a pneumotachometer placed at the endotracheal tube to account for the volume of delivered gas lost due to the distensibility of the ventilator circuit)? What about specialized populations, specifically those with congenital heart disease and small infants?

Unfortunately, we are left with the uncertainty on whether definitive $\mathrm{V}_{\mathrm{T}}$ data for pediatric ALI will be obtained in the future. Some have questioned the ethics of such a study. It is likely that the ethical and, of course, scientific, views will be based on the proposed study groups. Many would oppose the study of $6 \mathrm{~mL} / \mathrm{kg}$ versus $12 \mathrm{~mL} / \mathrm{kg}$ in pediatrics. But what about $4 \mathrm{~mL} / \mathrm{kg}$ versus $8 \mathrm{~mL} / \mathrm{kg}$ or $3 \mathrm{~mL} / \mathrm{kg}$ versus $6 \mathrm{~mL} / \mathrm{kg}$ ? Of importance to the study design is the concern about overlap as the potential study groups approach each other. Furthermore, what should be the age and weight cohorts for such a study? Finally, what about infants and children with normal lungs who are ventilated for a nonpulmonary reason? Unfortunately, the questions continue to outnumber the available answers.

The next topic addressed was that of PEEP management. Studies of adult ALI and ARDS have shown improvements in oxygenation, pulmonary compliance, and duration of ventilation, but no definitive change in mortality. ${ }^{20-22}$ Thus, the extrapolation of the available adult data to pediatrics becomes even less clear for PEEP management than for $\mathrm{V}_{\mathrm{T}}$ delivery.

Similarly, in the design of a pediatric PEEP study, many questions exist. Would it be possible to obtain an adequate sample size? Are the available PEEP- $\mathrm{F}_{\mathrm{IO}_{2}}$ tables from the ARDS Network study ${ }^{20}$ applicable to pediatrics, or are they too aggressive for infants and children? Would PEEP phobia inhibit a high PEEP approach in pediatrics? What about age and weight cohorts and patients with congenital heart disease? As mortality is unlikely to show a difference in a pediatric ALI study, what should be the primary end point? Finally, could such a PEEP study even be conducted until after the pediatric $\mathrm{V}_{\mathrm{T}}$ question is answered?

The issues and questions are similar for several other management aspects of pediatric ALI, including corticosteroid administration and fluid management strategies.
On the other hand, there are several areas in which pediatric data and experience may be extrapolated to the adult population. These include surfactant administration and extracorporeal membrane oxygenation (ECMO) for refractory ARDS. Despite the inherent differences between adult and pediatric patients, the sharing of data remains important. It must be stressed that such an approach should proceed with a careful assessment of the limitations of the available data for a specific management strategy.

\section{Noninvasive Ventilation of Premature Infants: Do We Really Need to Intubate?}

Invasive mechanical ventilation has been the hallmark strategy for supporting premature neonates with lung disease for nearly 4 decades. In the past decade, clinicians have witnessed a fundamental shift in ventilation practices among this patient population. As long as a neonate appears to have some spontaneous respiratory effort, nasal continuous positive airway pressure (CPAP) is generally initiated within the first few minutes of life. This approach is being implemented successfully in the delivery room or following surfactant administration and short-term invasive ventilation in the neonatal intensive care unit (NICU). However, approximately half of all neonates who are supported with CPAP still develop respiratory failure and require potentially injurious invasive ventilation.

Noninvasive ventilation (NIV) is a form of respiratory assistance that provides a greater level of respiratory support than CPAP and may prevent intubation in a larger fraction of neonates who would otherwise fail CPAP. With the inception of nasal airway interfaces, clinicians have ushered in many different forms of NIV in neonates, often with very little experimental data to guide clinical management. These NIV approaches include intermittent mandatory ventilation (IMV), neurally adjusted ventilatory assist (NAVA), "sigh" positive airway pressure (SiPAP), and high-frequency ventilation (HFV).

Rob DiBlasi began his discussion with the unique historical aspects of NIV and carefully reviewed the available NIV evidence related to the different forms of NIV being used in the NICU setting. ${ }^{23}$ It is interesting to note that neonatal NIV is not a new concept. In the early 1970s, clinicians reported unsuccessful NIV with an oronasal mask attached to an intermittent-positive-pressure-breathing machine or pediatric volume-cycled ventilator. This practice was dismissed in the NICU following several reports of higher incidence of gastrointestinal perforation and head molding and consequent cerebellar hemorrhage.

Today the most widely used and studied form of NIV is nasal IMV with a conventional ventilator in the pressure control mode. The most common patient interface is binasal short prongs. It has been postulated that nasal prongs provide a natural lung-protective pressure-relief valve 
through the leaky nasal airway interface and the oropharynx, which may prevent excessive pressure transmission to the distal airways or gastrointestinal tract. Compared to CPAP, NIV with IMV generally provides a higher mean airway pressure, which provides more lung recruitment, more consistent $\mathrm{V}_{\mathrm{T}}$, lower work of breathing, and better gas exchange. Additionally, IMV can evoke a beneficial "sigh" maneuver in neonates, which may help recruit areas of microatelectasis or airway collapse and might reduce apnea. However, DiBlasi proposed that IMV is more likely to prevent apnea than to support a patient who has persistent apnea.

In his review of the recently available clinical evidence, DiBlasi reported that IMV is being implemented in premature neonates using the following strategies:

- Post-extubation following long-term invasive ventilation

- Post-extubation, following surfactant replacement and short-term invasive ventilation

- As an initial form of support (prior to intubation) in neonates with signs of respiratory distress

Recent meta-analyses and several RCTs found that, compared to CPAP, neonates supported with IMV had a lower incidence of apnea and bronchopulmonary dysplasia (BPD), and better gas exchange, with no greater risk of cerebellar hemorrhage or gastrointestinal perforation. Larger studies are needed to determine the best timing for this exciting new NIV strategy in neonates, and to determine whether patient-triggered IMV is preferable to machine-triggered IMV during NIV in neonates.

NAVA is an exciting ventilation strategy that was recently introduced to the NICU. NAVA requires measurement of the electrical activity of the diaphragm by an indwelling esophageal catheter equipped with electrodes. The diaphragm signal determines the timing and magnitude of inspiratory pressure delivery during spontaneous breathing. There are many proposed advantages of NAVA over IMV, but only 2 reports have been published, from short-term studies that evaluated patient-ventilator interaction during noninvasive NAVA. Although those data are exciting, larger studies are needed to assess whether this expensive and labor-intensive ventilation strategy is desirable over other forms of NIV.

SiPAP differs from other forms of NIV in that it allows the neonate to breathe continuously at 2 separate CPAP levels. In combination with spontaneous breathing, these "sigh breaths" are intended to recruit unstable air spaces, maintain end-expiratory lung volume, and avoid apnea. Several small clinical studies have found that neonates on SiPAP had less need for invasive ventilation than those on CPAP. However, additional clinical research is needed on SiPAP.
Over the past 5 years, nasal HFV has been used more commonly in clinical practice as a form of NIV. Unfortunately, HFV is such a new form of NIV that very few data are available to suggest a long-term management strategy in neonatal patients. Short-term observational studies have suggested better gas exchange with HFV, compared to CPAP. A large RCT of HFV, with an appropriate nasal airway interface, is needed.

During the discussion, several of the faculty felt strongly that the major limitation of NIV is the lack of appropriate nasal airway interfaces, for both neonates and larger pediatric patients. It was agreed that better interfaces, designed to reduce nasal injury and to extend NIV to larger pediatric patients, should be a major focus of research and product development.

The prevailing message from this intriguing presentation was that there is a large population of neonatal patients who cannot be supported by CPAP alone. The clinical consensus is that invasive ventilation should be avoided if possible. In the not so distant future, clinicians may rely more on other forms of NIV, using IMV, NAVA, SiPAP, and HFV in premature neonates who fail CPAP or when weaning from invasive ventilation. While NIV is still early in its inception, clinicians will need further clinical research to determine the best form to use in their patient population.

\section{Neonatal Invasive Mechanical Ventilation}

Melissa Brown provided an overview of advances in neonatal ventilators and discussed whether these proposed improvements have resulted in better care of the premature neonate. ${ }^{24}$ The current trend in the NICU is to use NIV whenever possible, although invasive ventilation is often necessary for pre-term neonates with lung disease.

Invasive ventilation has been provided with neonatalspecific pressure ventilators for nearly 4 decades, but there are many new ventilator modes and strategies to assist with optimizing mechanical ventilation and preventing ventilator-induced lung injury. Patient-triggered ventilation with flow-triggering is one of those advances, as compared to the earlier generation of machine-triggered breaths. Synchronized IMV, continuous mandatory ventilation, and pressure support ventilation are the most common forms of patient-triggered ventilation. In short-term studies in premature neonates, patient-triggered ventilation had better patient-ventilator synchrony, better gas exchange, and less need for invasive ventilation than did IMV. However, no studies have shown that patient-triggered ventilation improves mortality or morbidity in premature neonates.

NAVA was recently FDA approved for invasive and noninvasive ventilation. NAVA had clinical benefits in short-term studies, but additional clinical trials are needed 
to determine NAVA's clinical efficacy and cost effectiveness.

New evidence suggests that volume-targeted ventilation modes provide better lung protection and better outcomes in premature neonates than do traditional pressure control modes. Several volume-targeted modes that provide accurate $V_{T}$ delivery despite a large endotracheal tube leak were recently introduced.

There continues to be debate about whether neonates should be managed invasively with HFV or conventional ventilation with a lung-protective strategy at birth. Based on several meta-analyses, HFV should be implemented early rather than as a rescue intervention, and with a strategy that optimizes lung recruitment. The majority of clinical trials have compared HFV to pressure control modes during conventional ventilation. Future trials enrolling premature neonates should compare HFV to conventional ventilation with volume-targeted modes. Because of a lack of available evidence, there is no consensus on whether highfrequency oscillatory ventilation is better than high-frequency jet ventilation for supporting neonates with respiratory distress syndrome (RDS).

Over the past decade, many new promising approaches to lung-protective ventilation strategies have evolved. Brown discussed recent animal and clinical research that has provided valuable insight on how best to manage neonates during conventional ventilation and HFV. The key to protecting the neonatal lung during mechanical ventilation is to optimize lung volume and limit excessive lung expansion. During conventional ventilation this can be achieved with appropriate PEEP, short inspiratory time $(0.25-0.5 \mathrm{~s})$, small $\mathrm{V}_{\mathrm{T}}(4-6 \mathrm{~mL} / \mathrm{kg})$, rapid respiratory rate, and permissive hypercapnia.

The future of neonatal invasive ventilation appears promising. New technologies are being developed at a rapid pace. These technologies are likely to improve the care for this delicate and vulnerable patient population. Management protocols are being devised with the best available experimental evidence. Widespread acceptance of new devices and approaches will continue to rely on data from well designed bench, animal, and clinical studies to better understand their role in the NICU environment.

\section{Advances in the Management of Pulmonary Hypertension}

Pulmonary hypertension (PH) is a relatively rare disease in infants and children, but occurs more frequently in neonates in the form of persistent $\mathrm{PH}$ of the newborn (PPHN). In general, PH is associated with substantial morbidity and mortality. PH is characterized by increased pulmonary vascular resistance related to increased pulmonary vascular reactivity, sustained pulmonary vasoconstriction, vascular remodeling, and/or vascular obstruction. $\mathrm{PH}$ is commonly diagnosed as a mean pulmonary arterial pressure $>25 \mathrm{~mm} \mathrm{Hg}$.

Although right-ventricular failure is a common final outcome in patients with $\mathrm{PH}$, the pulmonary vascular changes are diverse and relate to the specific etiology, which includes congenital heart disease, idiopathic PH, PH associated with pulmonary diseases, and (in neonates) PPHN. The pathophysiology of $\mathrm{PH}$ in children is multifactorial, complex, and not completely understood. Moreover, a minority of $\mathrm{PH}$ research is focused specifically on neonatal and pediatric patients.

Jeffrey Fineman, an established pediatric PH researcher, presented an inclusive review of PH etiologies, clinical manifestations, physiologic principles, and current and future therapies. ${ }^{25}$ Based on recent findings, several physiologic pathways are common to most forms of $\mathrm{PH}$. These discoveries have led to a better understanding of the role of systemic and pulmonary vascular endothelial cells and have elaborated several factors that mediate vascular smooth-muscle cell relaxation and constriction. Thus, the majority of approved and emerging PH therapies are targeted on manipulating endothelial-derived factors and/or their signaling pathways.

One area of active research is the mechanisms of endothelial injury and dysfunction in $\mathrm{PH}$, which include alterations in mechanical forces (such as increased pulmonary blood flow associated with congenital cardiac defects, and flow velocities altered by luminal narrowing) resulting in increased vascular wall shear stress, hypoxia, oxidative stress, and inflammation. Additional factors that contribute to endothelial injury in some patients include infection, such as human immunodeficiency virus or schistosomiasis, and injury from drugs or toxins. ${ }^{26-28}$

Endothelial-derived factors such as nitric oxide (NO), prostaglandin I2, and endothelin 1 are integral in the processes that regulate vascular smooth-muscle tone. Endothelial injury and dysfunction contribute to alterations in inflammatory cascades, growth factors, and transcriptional factors, which are increasingly recognized as key mediators of the vascular remodeling associated with muscle cell tone. It is also clear that underlying genetic abnormalities participate in the development of $\mathrm{PH}$ in some patients.

The clinical goals of PH therapy depend on the patient's clinical situation, and can include prevention and/or treatment of pulmonary vasoconstriction, support of right-ventricular function, treatment of the underlying disease (if possible), and promotion of regressive remodeling of structural pulmonary vascular changes. Of course, these aims are interrelated, but certain therapies may target one aim more than another in a given patient.

Historically, metabolic and respiratory alkalinization has been a part of the initial resuscitation strategy in patients with PH. However, long-term application of those therapies can be detrimental. For example, aggressive hyper- 
ventilation and oxygenation can promote secondary lung injury in neonates with PPHN. As such, gentle ventilation with mild permissive hypercapnia, lower $\mathrm{F}_{\mathrm{IO}_{2}}$, and inhaled nitric oxide (INO), with or without HFV, have become accepted practice for neonates with severe PPHN.

The most widely used $\mathrm{PH}$ therapies alter one of the 3 endothelial signaling cascades: nitric oxide/cyclic guanosine monophosphate (NO-cGMP), prostaglandin I2, and endothelin-1. INO is the best studied and most widely used agent for acute selective pulmonary vasodilation. Although INO is FDA-approved only for neonates with hypoxemic respiratory failure, it is frequently being used off-label to treat other forms of $\mathrm{PH}$, and for diagnosis.

Sildenafil is a phosphodiesterase-5 inhibitor that augments NO-cGMP signaling pathways and results in pulmonary vascular relaxation by inhibiting the degradation of cGMP. RCTs that included pediatric patients found oral sildenafil effective in patients with chronic PAH. Despite limited data, the use of sildenafil in infants and children with $\mathrm{PH}$ after cardiac surgery is increasing. Sildenafil may have benefits equal to INO, and may assist with weaning from INO in some patients.

$\mathrm{L}$-arginine is decreased in neonates with PPHN and adults and children with $\mathrm{PH}$ related to various maladies. Like INO and sildenafil, L-arginine augments the NO-cGMP cascade. Ongoing clinical research suggests that L-arginine helps reduce the effects of PH in neonates with PPHN, patients with sickle cell disease, and following cardiopulmonary bypass in patients with cardiac defects.

Despite the many recent advances in therapy, prostanoids, such as intravenous prostaglandin I2 (epoprostenol), remain the best-proven and most effective therapy for chronic PH. Treprostinil is another prostaglandin I2 analog that was recently approved for intravenous administration. Data are not available to compare the efficacy of intravenous epoprostenol to treprostinil. To achieve selective pulmonary vascular relaxation, various investigations have focused on delivering prostanoids via inhalation. Iloprost and trepronistil are prostaglandin I2 analogs that are FDAapproved for inhalation. Recent data suggest that iloprost reduces pulmonary vascular resistance as much as does INO, and at lower cost.

Newer drugs are aimed at inhibiting endothelin- 1 and endothelin-A, which are signaling pathways that, when antagonized, promote vasodilation. Several exciting combination therapies using these drugs suggest that there may be some benefit to using them together. Fineman mentioned several other prospective drugs that are currently being tested in patients with $\mathrm{PH}$, including endothelialbased therapies that use endothelial progenitor cell infusions, superoxide dismutase, vasoactive intestinal peptide, adrenomedullin, calcium-channel blockers, and anticoagulation therapies, which may show great promise for patients with PH. Statins and several other chemotherapies used for leukemia are also now being used in patients with $\mathrm{PH}$, to address pulmonary vascular remodeling.

Since no single drug or drug combination has been found that completely alleviates the symptoms of increased pulmonary vascular resistance in patients with $\mathrm{PH}$, an important goal is to support the right ventricle, by reducing right-ventricular afterload (ie, reduce pulmonary vascular impedance), to optimize right-ventricular blood volume, to augment right-ventricular contractility, and to maintain systemic vascular resistance with proper volume loading and inotropic support. Atrial septostomy is a surgical (or cardiac catheterization) intervention for managing chronic $\mathrm{PH}$, and has been advocated to allow for decompression of the right ventricle by promoting right-to-left shunting. Isolated lung transplantation, as opposed to requisite heart-lung transplantation, is now a viable option for some children with end-staged $\mathrm{PH}$.

Over the past several decades, fundamental advances in pulmonary vascular biology have been translated into effective therapies that have improved the quality of life and prolonged survival for neonates, infants, and children with $\mathrm{PH}$. Many novel therapies show great promise for patients with $\mathrm{PH}$.

\section{Inhaled Medical Gases: More to Breathe Than Oxygen}

Inhaled medical gases are an integral part of the management of critically ill patients. Mike Gentile provided an overview of several of the available medical gases-beyond oxygen. ${ }^{29}$ Complementing Fineman's discussion, this presentation began with an overview of INO. Gentile noted that this topic is still actively discussed and studied, despite the availability of over 100,000 papers on nitric oxide and over 4,000 publications specifically focused on INO. The medical importance of INO as a selective pulmonary vasodilator rests on the fact that it can be delivered as a gas directly to the pulmonary circulation, without systemic adverse effects.

The data on INO in PPHN are considered definitive by most. Beyond its FDA-approved indication for PPHN, INO is administered off-label for several other conditions, including prematurity, acute lung injury, and congenital heart disease.

Clinical trials have delivered INO to premature infants ( $\leq 34$ weeks gestational age) for the treatment and prevention of chronic lung disease and for supporting gas exchange in premature infants with RDS. ${ }^{30-33}$ Gentile concluded that, at present, INO for premature infants is not supported by the medical literature, nor can INO be recommended for patients with ARDS, except as a potential bridge to ECMO when improved oxygenation and overall clinical stability are needed for a short period. Despite the belief that INO may improve outcomes for children and 
adults with ALI and ARDS, by improving oxygenation and thus allowing decreased ventilator settings and decreasing the risk of ventilator-induced lung injury, the available data are not supportive. ${ }^{34-40}$

Gentile speculated that future INO research will evaluate the role of INO in lung injury and inflammation and other disease states. As more knowledge is gained about the interaction of INO and the human body, new and promising therapies may emerge to treat cardiopulmonary diseases. However, alternative intravenous, inhaled, and enteric alternatives to INO will probably emerge with increasingly important roles as selective pulmonary vasodilators.

Gentile then discussed heliox. Unlike INO, the data for heliox in patients with airway obstruction are limited. There have been no definitive, large-scale RCTs on heliox. At present, clinicians must rely on the data from mostly small, single-center clinical studies and on our understanding of the physics of gas exchange and the physiology of airway obstruction. Heliox is a safe and rapidly acting gas that reduces airway resistance and work of breathing and improves gas exchange in various respiratory conditions. The benefit of heliox lies solely in its low density. Published research on heliox dates back over 70 years and suggests that it may be useful in situations of substantial airway obstruction.

Although the literature on heliox contains both positive and neutral studies, there have been no reported adverse effects, which is not unexpected, because heliox is biologically inert. Potential complications of heliox are related to technical aspects of heliox delivery. The clinician who administers heliox must understand how heliox's lower density gas affects the functioning of respiratory equipment.

Although the use of inhaled anesthetic is commonplace in surgery, its use in the ICU remains very limited. Potential ICU applications include as an adjunct to sedation management, and the treatment of refractory status asthmaticus and status epilepticus. Despite potential efficacy for these conditions, a variety of reasons exist for the lack of its use in the ICU, including limited equipment availability, lack of clinical experience and expertise among intensivists, and unknown consequences of long-term exposure to various organ systems. Thus, there are no clear guidelines for inhaled anesthetic in the ICU. Special considerations include appropriate equipment for inhaled anesthetic delivery, gas scavenging systems to protect staff, and appropriate monitoring systems and staff training to ensure patient safety. These very substantial limitations are likely to continue to greatly limit the use of inhaled anesthetics in the ICU.

A specialized and vulnerable population that has been the focus of a longstanding debate involving inhaled medical gases are infants with single-ventricle physiology (ie, hypoplastic left-heart syndrome). Historically, the goal in the management of these complex infants has been to increase pulmonary vascular resistance to reduce pulmonary blood flow and thus augment systemic blood flow. The 2 methods to increase pulmonary vascular resistance have been to administer either carbon dioxide or nitrogen via the inspiratory limb of the ventilator. Carbon dioxide is a potent pulmonary vasoconstrictor, whereas supplemental nitrogen creates a hypoxic gas mixture that promotes pulmonary vasoconstriction. These approaches have been well reported in the medical literature. ${ }^{41-45}$ To optimize patient safety, comprehensive monitoring systems and carefully considered policies and procedures should be in place before utilizing either of these inhaled therapies.

It should be stressed that the use of hypercarbic or hypoxic gas therapy in infants with single-ventricle physiology has become increasingly rare with advances in the preoperative and postoperative management of these infants. Preoperatively, most of these infants can balance their pulmonary/systemic blood flow ratio without intervention. Postoperatively, advances in medical and surgical management have made hypercarbic or hypoxic gas therapy rarely indicated.

Gentile intrigued the conference attendees with his description of 2 gas therapies not yet ready for clinical use: inhaled carbon monoxide (CO) and inhaled hydrogen sulfide $\left(\mathrm{H}_{2} \mathrm{~S}\right)$. Recent investigations discovered that low-concentration inhaled $\mathrm{CO}$ is an important vascular paracrine factor. Models of ventilator-induced lung injury, aspiration, hyperoxia, and ischemia/reperfusion injury found that inhaled $\mathrm{CO}$ benefited several pulmonary disorders. ${ }^{46-50}$ Over the past few years, the role of $\mathrm{H}_{2} \mathrm{~S}$ as a physiologic messenger has become a focus of scientific investigation. Although the preclinical data describing $\mathrm{H}_{2} \mathrm{~S}$-induced "suspended animation" are promising, extensive further investigation is required prior to any clinical application for the management of shock and other critical illnesses.

Gentile concluded that inhaled medical gases have become integral to the care of neonatal and pediatric patients. To improve gas exchange and to provide physiologic benefit while other therapies are explored or implemented, inhaled gases will continue to play an integral role in the respiratory care of critically ill patients. INO (beyond the neonatal population) and heliox will probably see variable use as ICUs interpret and apply the available data differently. Inhaled anesthetic will continue to be used only rarely in the ICU. It is too early to predict whether $\mathrm{CO}$ and $\mathrm{H}_{2} \mathrm{~S}$ will prove beneficial in critically ill patients.

\section{Resuscitation in the Delivery Room: Lung Protection From the First Breath}

Our focus then moved to the delivery room. Resuscitation is required in approximately $10 \%$ of the more than 100 million infants born annually worldwide. The resus- 
citation techniques, including positive-pressure ventilation and supplemental oxygen, can have adverse pulmonary effects, and increasing attention has been applied to providing lung protection from the first breath.

Tom Wiswell, a pioneer in neonatal resuscitation, provided an insightful overview of the available evidence on delivery room management to avoid lung injury. ${ }^{51}$ Based on animal and human research, several mechanisms have been described that could injure lungs during our newborn resuscitative efforts..$^{52}$

Volutrauma refers to lung overdistention caused by assisted ventilation. Inflammation and physical damage may be consequences. Even a few large manual breaths can cause injury, and high $\mathrm{F}_{\mathrm{IO}_{2}}$ can be toxic to the lungs. High $\mathrm{F}_{\mathrm{IO}_{2}}$ produces oxygen free radicals such as superoxide, hydrogen peroxide, and hydroxyl radical. Several studies of outcomes in neonates resuscitated with $\mathrm{F}_{\mathrm{IO}_{2}}$ of 1.0 versus room air in the delivery room setting have indicated that using room air as the starting point during resuscitation resulted in similar short-term outcomes to $100 \%$ oxygen.

Sorely lacking in all of the available studies are outcome data on major morbidities: chronic lung disease, persistent $\mathrm{PH}$, intracranial hemorrhage, cerebral-whitematter injury, necrotizing enterocolitis, patent ductus arteriosus, and retinopathy of prematurity. Based on several reviews of the available evidence, there is no consensus on what $\mathrm{F}_{\mathrm{IO}_{2}}$ to use initially during resuscitation, and the available guidelines have wavered. The 6th edition of the Textbook of Neonatal Resuscitation (recently published by the Neonatal Resuscitation Program in conjunction with the American Academy of Pediatrics and the American Heart Association) includes a table of target pre-ductal saturations to aim for during resuscitation..$^{52}$ If an infant's oxygen saturation is below the lower limit at specified time points, it is recommended that oxygen should be administered via a blender, for an $\mathrm{F}_{\mathrm{IO}_{2}}$ sufficient to achieve the target $\mathrm{S}_{\mathrm{pO}_{2}}$. In term infants the Textbook of Neonatal Resuscitation advocates, but does not mandate, using room air at the initiation of resuscitation.

Although there are many anecdotal reports that the routine use of CPAP or PEEP in the delivery room in preterm infants appears promising, the data are less than compelling; the available data indicate that neither CPAP nor PEEP in the delivery room affect the major outcomes death and BPD. And in term-gestation infants there are virtually no scientific data on delivery room CPAP or PEEP. As such, it has been suggested that the use of CPAP or PEEP should reflect local expertise and preferences. Sustained inflations for the first several breaths of manual ventilation is a popular method to assist the formation of functional residual capacity during resuscitation. Although sustained inflations appears to be an intriguing method of opening the lung, Wiswell does not believe the available data sup- port the use of sustained inflations during delivery room resuscitation.

Surfactant replacement therapy is frequently administered in the delivery room to premature neonates. There is currently no consensus reported by the Neonatal Resuscitation Program on the timing of surfactant administration in the delivery room, so clinicians have to make their own decisions on the timing of surfactant therapy in preterm infants.

Prevention of meconium aspiration syndrome has long been a goal in the delivery room. Approximately $12-15 \%$ of all newborn infants are born through meconium-stained amniotic fluid, and 3-10\% of those develop meconium aspiration syndrome. Meconium inactivates endogenous surfactant and can directly injure lung parenchyma and cause necrosis and hemorrhage. Multiple delivery room maneuvers have been tried to prevent meconium from entering the trachea and lower airways. For a 25 -year period starting in the mid-1970s, the routine teaching was to intubate all meconium-stained infants, to suction the trachea. Early evidence suggested intrapartum (ie, prior to delivery of the thorax) suctioning of the oropharynx and nasopharynx of meconium-stained infants, but, based on the findings from several large studies, it is now suggested that no data clearly support that approach. Wiswell suggested that a vigorous meconium-stained infant does not need intubation or tracheal suctioning, whereas a non-vigorous meconium-stained infant should be suctioned via an endotracheal tube. If the intubation attempt is prolonged and unsuccessful, bag-mask ventilation should be considered, particularly if there is bradycardia.

Guidelines for neonatal resuscitation continue to be modified based on the best available evidence. According to Wiswell's review, there are inconsistencies across delivery room care that remain to be addressed. Hopefully, well designed future studies with large numbers of infants will help eliminate those inconsistencies.

\section{Surfactant: Neonates and Beyond}

Surfactant replacement therapy reduces surface tension in the lungs, improves gas exchange and compliance, increases functional residual capacity, and reduces work of breathing. Clinical trials in the 1980s unequivocally demonstrated that exogenous surfactant improved survival and decreased morbidity in premature neonates with RDS. In the first year after FDA approval of surfactant therapy there was a notable decrease in neonatal mortality, primarily related to improved survival of preterm infants. Thus, surfactant replacement has become routine for the prevention and treatment of infant RDS and other causes of neonatal ALI. The role of surfactant in lung injury beyond the neonatal period, however, has proven to be a more complex topic. 
Doug Willson focused much of his presentation on the role of exogenous surfactant for ALI and ARDS in larger infants and children. ${ }^{53}$ Willson described the current situation with surfactant administration for this patient population. Additional clinical trials are needed before widespread use of surfactant beyond the neonatal period can be routinely recommended.

Relative surfactant deficiency, dysfunction, and inhibition all contribute to the disturbed physiology seen in ALI and ARDS, so exogenous surfactant seems a plausible therapy, but it has been less effective in pediatric and adult ALI/ARDS than in RDS, where simple deficiency is causative. The apparent lack of efficacy in the non-neonatal population may relate to inadequacy of pharmaceutical surfactants, insufficient dosing or drug delivery, poor drug distribution in the lung, or simply an inability of the drug to substantially impact the underlying pathophysiology of ALI/ARDS (ie, surfactant dysfunction and inhibition). There are several different surfactant formulations (eg, synthetic preparations and natural surfactants derived from lavaged animal lung or processed animal lung tissue), and it is unclear which of these will prove most useful in pediatric ALI/ARDS patients.

Both animal and human studies suggest that direct types of ALI (eg, aspiration, pneumonia) are more responsive to surfactant therapy than the indirect types (eg, sepsis, pancreatitis). A potential benefit of early exogenous surfactant may be to minimize or ameliorate ventilator-induced lung injury. Improved compliance and better alveolar aeration at lower pressure and $\mathrm{V}_{\mathrm{T}}$ from early surfactant administration may stop the vicious circle of ventilator-induced injury (lung injury requires increased ventilator support, which causes further lung injury), but additional data are required to confirm this speculation.

Willson reviewed the pertinent animal data on surfactant in ALI/ARDS. Animal studies, though only descriptive from a scientific perspective, have helped to elucidate surfactant efficacy and administration, and remain indispensible for investigating and optimizing surfactant delivery, dosing, timing of therapy, surfactant composition, and lung distribution. Questions of safety and clinical efficacy ultimately require clinical studies. A substantial number of uncontrolled and controlled trials have documented clinical benefits from surfactant therapy in term infants, children, and adults with acute respiratory failure. Despite those studies' relatively small size and mixed findings, the sum of their findings is that there are several specific indications where the evidence for surfactant therapy in acute pulmonary injury is promising. However, the experience with surfactant in adults with ALI/ARDS has been much less encouraging than in younger patients.

A recent blinded controlled pediatric ALI/ARDS study by Willson et $\mathrm{al}^{8}$ found that calfactant (versus placebo) was associated with immediate oxygenation improvement and a significant survival advantage. In a post hoc analysis, the benefits of surfactant therapy were confined to the 98 patients who had direct pulmonary forms of ALI/ARDS, which prompted a larger prospective study, the Calfactant for Direct Acute Respiratory Distress Syndrome (CARDS) trial (http://clinicaltrials.gov/ct2/show/NCT00682500), which was a prospective masked RCT of calfactant versus placebo in children and adults with ALI/ARDS from direct lung injury. The study was carried out in more than 30 centers in the United States, Canada, Korea, Israel, Australia, and New Zealand. The data analysis is incomplete, but, unfortunately, both the adult and pediatric arms of the study have been stopped because of futility. The reasons for the failure of the CARDS trial after the success of the previous trial by Willson et $\mathrm{al}^{8}$ remain to be elucidated.

Willson explained that ALI/ARDS is multi-faceted and complex, and has great potential for combination therapies. The use of exogenous surfactant in conjunction with other modalities to simultaneously attack different aspects of ALI/ARDS pathophysiology may prove to be synergistic-not unlike the use of combination chemotherapy for various types of cancer. The combined use of drugs, including surfactant, low- $\mathrm{V}_{\mathrm{T}}$ ventilation, and careful fluid management, may prove beneficial.

In summary, the jury is still out with respect to the use of surfactant therapy in children and adults with ALI/ARDS. Although several negative studies exist, we must consider the perspective of history: the first trials of surfactant therapy for neonatal RDS were unsuccessful because they used ineffective surfactants and delivery methods. It was more than 2 decades after surfactant deficiency was first suggested as the cause of RDS before successful treatment of premature infants with exogenous surfactant occurred. Finding the most successful surfactant interventions, delivery methods, and combination therapies for ALI/ARDS may simply require more time. Further study, and perhaps the development of more robust pharmaceutical surfactants, offer promise that exogenous surfactant will find a place in our armamentarium for the treatment of ALI/ ARDS.

\section{Asthma 2015 and Beyond}

Tim Myers provided a very comprehensive discussion of status asthmaticus, including some provocative thoughts for the future. ${ }^{54}$ Unlike many of the other topics presented, the field of pediatric asthma has substantial definitive data to guide clinical management. ${ }^{54}$

Any discussion of asthma must involve the epidemiology and pathophysiology. There is a clear disproportion among the general population; however, it remains unclear whether this finding is predominantly genetic or environmental. Myers concluded that, "though science has not found strong support for either hereditary or hygiene fac- 
tors, these theories have not been invalidated either. The pathogenesis of asthma is probably related both to genetic predisposition and to over-exposure or under-exposure to viruses or other environmental factors." ${ }^{55-62}$ As a related topic, we must consider the various phenotypes of asthma, as improved phenotype definition may help in identifying risks factors for an exacerbation in a specific patient as well as individualized management approaches. Myers noted that asthma management guidelines are increasingly recommending phenotype-specific treatment.

Diagnosis, assessment, and monitoring were another clear focus of this discussion of pediatric asthma. Of importance, asthma severity has been declining, and this improvement may be partially attributed to severity-based treatment strategies. ${ }^{63}$ Other potential explanations for this improvement trend include increased patient adherence to maintenance therapy, advances in pulmonary monitoring, and changes in management strategies. Myers noted that antihistamines, xanthines, and cromones have virtually disappeared from the routine asthma armamentarium. The bases of today's pediatric asthma management are chemically refined derivatives of adrenaline and cortisone. The newer formulations of these medications are much improved in terms of adverse effects, but are still not perfect. Inhaled corticosteroids have become the cornerstone of the management of persistent asthma. A small minority of the people with asthma account for the majority of asthma healthcare utilization, so new treatments and approaches to this refractory sub-population is a high priority.

Myers concluded with a very important discussion on asthma education. He stressed that the effects of environmental change in the prevention of asthma exacerbations is intrinsically linked to education. Potential approaches include Internet programs for home use, school-based interventions that focus on self-management, and coalitions and partnerships to focus on community-wide changes. A supplement to the March 2009 issue of Pediatrics ${ }^{64}$ described the need for standardization and collaboration, the use of childhood asthma as a model to test a performancebased system, and the need for evidence-based interventions that tailor care to individuals as well as communitywide characteristics.

\section{Aerosol Delivery: An Old Topic With New Data}

Bruce Rubin continued the discussion on airway management and disease with a lively discussion on new drugs and delivery devices for neonatal and pediatric patients. ${ }^{65}$ Choosing the appropriate aerosol device, monitoring patient technique, assessing the response to therapy, and evaluating potentially better drug formulations are important activities in which the respiratory clinician must remain competent. Keeping up with the rapidly growing body of evidence on devices, drugs, and delivery techniques can be a daunting task. An additional challenge is critically reviewing the evidence and determining whether these, often costly, new therapies will actually improve drug delivery and outcomes in patients.

Rubin began by discussing the more promising pediatric devices that might make drug delivery more precise, less wasteful, and easier for the youngest and most incapacitated of patients. He elaborated on some of the newer techniques used to deliver inhaled medications and even dispelled a few myths about aerosol delivery to infants and toddlers. Infants and toddlers who do not tolerate aerosol delivery via mask will not do better with the blow-by approach, as drug delivery to the distal airways is negligible with blow-by. One available device delivers aerosol to the small child via a hood that covers the child's head while the child is asleep. There is also an innovative system that incorporates a pacifier into an aerosol mask, soothing the child who sucks on the pacifier to allow the mask to rest on the face as the infant inhales through the nose. A key point is that aerosol therapy works best if a patient is breathing quietly.

Industry leaders have ushered in a new line of smallvolume liquid inhalers. The Respimat Soft Mist Inhaler is a disposable, spring-loaded device that provides a highvelocity bolus of medication that improves coordination and medication delivery and may be easier to use than a metered-dose inhaler with spacer. The Aeroneb Go, Omron MicroAir, and Pari eFlow are examples of new vibrating-mesh aerosol devices that can be optimized for different drugs by adjusting the pore size of the mesh, the aerosol chamber size, the reservoir size, and the output rate. The AeroEclipse is a breath-actuated nebulizer that delivers aerosol only during inspiration and, thus, may be most useful in terms of conserving drug. This device is triggered by a valve, and it remains unclear whether very small patients can trigger such devices effectively.

Breath-controlled nebulizers have servo-controlled medication delivery that is based on the measured inspiratory flow pattern, to assure that drug is being delivered to the small airways. Adaptive aerosol delivery is a multiplebreath technology developed by Philips-Respironics; the device is preprogrammed with the inhalation dose, and a computer "learns" how the patient is breathing and adapts to changes in the breathing pattern, averaged over several breaths. These highly efficient devices are costly and may be better suited for delivering expensive drug formulations, so their role in delivering less expensive drugs is less clear. Additional studies are required to determine their clinical efficacy, efficiency, and cost-effectiveness.

Inhaled antibiotics and antivirals are increasingly being used to treat difficult or persistent airway infections, ventilator-associated pneumonia, and drug-resistant tuberculosis. Inhaled liquid antibiotic formulations can provide a high concentration of antibiotic to the distal airways, po- 
tentially with fewer adverse effects than systemic (ie, intravenous) antibiotic administration. Many new and exciting inhaled antimicrobials are being developed for aerosol use, including quinolones (eg, ciprofloxacin and levofloxacin), aminoglycosides (eg, gentamicin and neomycin), and antifungal agents. Several novel inhaled powder drugs composed of engineered particles were also presented. These powdered antibiotic preparations are composed of potent particles that may prove to be more useful than liquid preparations in patients with persistent airway infections.

Rubin discussed anti-inflammatory drugs for patients with cystic fibrosis, as well as inhaled peptides (eg, granulocyte-macrophage colony-stimulating factor) for treating pulmonary alveolar proteinosus. Other large peptides for airway delivery might include gene-transfer therapy with complimentary DNA delivered as an aerosol in a vector package to affected cells. This gene-transfer-therapy strategy is under development for cystic fibrosis. There are a number of medications available to treat $\mathrm{PH}$, including drugs that increase nitric oxide, inhibit endothelin, or activate phosphodiesterase. Among these are the prostacyclin analogues epoprostenol and iloprost, which are well accepted as nebulized medications for treating severe PH. These drugs may provide a reduction in pulmonary vascular resistance similar to that of INO, but at lower cost. Prostacyclin analogues with longer half-life (eg, treprostinil) and controlled-release formulations are in clinical development.

There have been dramatic advances not only in the medications that are being administered via aerosol but in the devices available for aerosol delivery. The future for new devices and new drugs appears very promising.

\section{Airway Maintenance and Clearance: How to Stay Out of Trouble}

Brian Walsh presented a detailed discussion on airwayclearance techniques and physiology. ${ }^{66}$ Echoing the comments made by several of the earlier presenters, Walsh commented on the lack of definitive pediatric data. There are substantial hurdles to doing well designed RCTs of the effectiveness of airway-clearance techniques, although the potential pediatric study population for airway-clearance research is substantially larger than that for ALI. In studying airway-clearance techniques it is difficult, and in some cases impossible, to blind the intervention. Additionally, many of the techniques are affected by patient cooperation, which is greatly affected by patient age and the individual characteristics of each child. Also complicating this topic is that data are less easily extrapolated from adults to children. Children, as compared to adults, have a greater density of submucosal glands, more acidic mucus (which may lead to higher viscosity), more compliant chest wall, lower functional residual capacity, a greater tendency for airways to collapse, and fewer collateral airways.

Walsh reminded us of some of the basic principles of airway suctioning. Both closed and open suctioning will de-recruit lung: that is, negative pressure is negative pressure! Thus, suctioning a patient immediately prior to extubation may not be an ideal approach. ${ }^{67}$ Data are currently lacking to demonstrate that post-suctioning recruitment maneuvers are helpful. However, the recruitment maneuvers used in the studies may have been inadequate. ${ }^{68}$ Various airway-clearance techniques and strategies were reviewed, including insufflation-exsufflation, intrapulmonary percussive ventilation, mucolytics (eg, dornase alfa), bicarbonate as an airway buffer, and nebulized hypertonic saline. These approaches have been variably effective and are detailed in his review.

Airway maintenance should include supporting normal physiology, including appropriate humidity, promoting normal ciliary function, preventing airway collapse, encouraging a normal cough response, and maintaining the lung environment as normal as possible. When choosing an airway-clearance modality the clinician should ask: Is there a physiologic rationale for the therapy? Is the patient truly experiencing difficulty clearing secretions? If so, are retained secretions adversely affecting lung function? What are the potential adverse effects of the proposed airwayclearance therapy? Is the cost of the therapy reasonable for the expected benefit? What is the preference of the patient and his or her family?

Remember that, since we lack evidence that any airwayclearance technique is superior to another, patient or family preference should be an important consideration. Walsh concluded with an important take-home message, that airway-clearance practices need to evolve based on highquality research, so we can be sure that the techniques are not unnecessarily contributing to the high cost of healthcare or, more importantly, causing harm to patients.

\section{ECMO: Moving at the Speed of Light}

Heidi Dalton provided a state-of-the-art presentation on the life-saving strategy of ECMO for refractory cardiac and/or respiratory failure. ${ }^{69}$ Much has been learned from the ECMO experience of the over 45,000 patients in the Extracorporeal Life Support Organization registry (http:// www.elso.med.umich.edu).

The technological advances in the field of extracorporeal life support rival any other in neonatal-pediatric respiratory care over the past decade. The most important of these advances include centrifugal pumps, hollow-fiber oxygenators, and improved double-lumen venous cannulas. These technologies are now available for even the smallest of ECMO patients. Centrifugal pumps lower the risk of hemolysis, allow for smaller priming volumes, and 
decrease pump setup time. Hollow-fiber oxygenators have a lower resistance, can be bio-coated or heparin-coated, and also have smaller priming volume. The improved double-lumen venous cannulas have allowed more use of venovenous ECMO and, in some cases, percutaneous insertion.

Dalton reviewed some of the recent trends in neonatal and pediatric ECMO. One of the most striking recent findings was about the relationship between survival and preECMO duration of ventilation. Traditionally, one ECMO criterion was that the patient had received less than 7-10 days of substantial ventilator support prior to ECMO cannulation. However, recent data support a pre-ECMO duration of ventilation of up to 14 days. ${ }^{70}$ Other trends include the increasing use of ECMO in older children and adults, incidence of comorbidities in ECMO patients, use of veno-venous ECMO, and the use of ECMO during cardiopulmonary resuscitation. Note, however, that the data on long-term neurologic outcomes in patients who undergo ECMO during cardiopulmonary resuscitation are still preliminary.

As clinicians look to the future of ECMO, Dalton offered several speculations. An increase in the use of ECMO in adults should be anticipated in follow-up to the Conventional Ventilation or ECMO for Severe Adult Respiratory Failure (CESAR) study. ${ }^{71}$ Also likely are decreased sedation use, increased physical rehabilitation, and increased use of ambulatory ECMO. ${ }^{72}$ With the simplification of ECMO systems we soon may no longer need a 1:1 nurse and ECMO specialist to patient staffing ratio, which would improve resource allocation. ${ }^{73}$ Moving into the field of ethics, Dalton discussed the controversial use of ECMO for organ preservation. She clearly indicated that with regard to this application, ethical considerations have not yet caught up with technology.

Dalton concluded her presentation with a request to "reframe ECMO" and stressed that ECMO should not be a result of desperation, but should be another tool in the clinician's toolbox. ECMO is much less complex today than just a few years ago, and is becoming less complex every day. There is not much doubt that the field of extracorporeal life support will continue to expand at an impressive pace in terms of technology and potential population base.

\section{Disaster Planning for Pediatrics}

The final talk, on pediatric trauma, was given by Rich Branson. ${ }^{74} \mathrm{He}$ highlighted the deficiencies in preparedness for pediatric trauma throughout the country and abroad. This presentation highlighted the key differences between pediatric and adult patients, which require different disaster-planning strategies. Anatomically, children have a larger head-to-body ratio and therefore a greater likelihood of head injury. The greater surface area of a child relates to the potential for larger evaporative fluid losses. Other key differences are that children are inherently closer to the ground and are often found in close groups (eg, schools). When adult clinicians manage pediatric patients, we must remember that normal vital signs not only differ by age and weight but are affected by anxiety and fever, so normative vital-sign charts may be difficult to apply to an individual pediatric patient.

Although many worry about mass disasters and focus planning on these rare scenarios, Branson eloquently stressed the importance of the " $n$ of 1 " disaster, meaning that one critically ill child in an adult clinical environment that is not equipped for pediatrics could quickly become a disaster. Adult hospitals and emergency departments are often not child-friendly because the personnel may lack pediatric-specific training, child-size equipment, pediatric dosing recommendations, or pediatric triage tools. Additionally, few general hospitals provide training in pediatric emergencies, and less than $6 \%$ possess a full complement of child-appropriate equipment, medications, and supplies. ${ }^{75-77}$ Branson discussed whether sick children can be managed as adult patients, and said that the answer depends on the specific hospital, the child's age and weight, and the pathophysiology of the disease or injury.

The bottom line recalls once again the clinical adage that children are not simply small adults. Although children make up approximately $22 \%$ of the United States population, pediatrics is grossly underrepresented in disaster planning. Children have unique physiology and pathophysiology, compared to adult patients/victims. There is clearly an urgent need for increased pediatric representation in local, regional, and national disaster preparedness programs.

As a nation, we must invest in increased capacity of our emergency system; promote consistent day-to-day pediatric readiness with routine education programs and training; build on the strength of existing systems; improve pediatric readiness of emergency response teams; and remove barriers to study, authorization, and deployment of pediatric-specific equipment, supplies, and medications. Additionally, it is essential that the appropriate agencies assure that shelters are child-friendly and must immediately improve child identification and family-reunification programs. Branson concluded by saying that children clearly need more of a national voice in disaster preparedness.

\section{Conclusions}

It is clear that we need additional neonatal and pediatric-specific RCTs on all of the topics presented. Although we have definitive data in some areas, in many other areas of neonatal-pediatric respiratory care the questions still greatly outnumber the available answers. When consider- 
ing the various topics discussed we must always consider the balance between safety and efficacy. When safety data exist without efficacy data, uncontrolled variables include the knowledge, experience, and support available in an individual patient care environment.

The primary goals of this Journal Conference were to review the available data, technology, and practice patterns for a variety of neonatal-pediatric topics, and, more importantly, to share thoughts and speculations for the future of the field. We hope the information presented and discussed will enable clinicians to make better-informed decisions. The topics chosen were intended to provoke thought, stimulate discussion with speculation for the future, and encourage further rigorous scientific investigation. The first and second goals have been at least partially achieved. It is our hope that this Journal Conference will lead to the accomplishment of our third goal in the near future.

As stated at the outset, we are extremely grateful to the contributing faculty of the 47th ReSPIRATORY CARE Journal Conference for their dedication, expertise, enthusiasm, and, of course, willingness to share their tremendous knowledge and experience. The success of this conference was due to the outstanding panel of contributing experts. We also very much appreciate the support the entire staff of ResPiratory CARe, along with the American Respiratory Care Foundation, for their assistance with and sponsorship of this very important Neonatal-Pediatric Journal Conference. This was truly an outstanding experience for all of the participants. We are hopeful that this issue of RESPIRATORY CARE has conveyed the excellent presentations and discussions among the panel of experts and will help clinicians to further improve the respiratory care of their patients.

\section{REFERENCES}

1. Curley MA. Respiratory research in the critically ill pediatric patient: why is it so difficult? Respir Care 2011;56(9):1247-1254; discussion 1254-1257.

2. Marshall JC, Cook DJ. Investigator-led clinical research consortia: The Canadian Critical Care Trials Group. Crit Care Med 2009;37(1 Suppl):S165-S172.

3. Curley MA. Prone positioning of patients with acute respiratory distress syndrome: A systematic review. Am J Crit Care 1999;8(6): 397-405.

4. Curley MA, Thompson JE, Arnold JH. The effects of early and repeated prone positioning in pediatric patients with acute lung injury. Chest 2000;118(1):156-163.

5. Curley MA, Hibberd PL, Fineman LD, Wypij D, Shih MC, Thompson JE, et al. Effect of prone positioning on clinical outcomes in children with acute lung injury: A randomized controlled trial. JAMA 2005;294(2):229-237.

6. Randolph AG, Meert KL, O'Neil ME, Hanson JH, Luckett PM, Arnold $\mathrm{JH}$, et al. on behalf of the Pediatric Acute Lung Injury and Sepsis Investigators Network. The feasibility of conducting clinical trials in infants and children with acute respiratory failure. Am J Respir Crit Care Med 2003;167(10):1334-1340.
7. Randolph AG, Wypij D, Venkataraman ST, Hanson JH, Gedeit RG, Meert KL, et al; Pediatric Acute Lung Injury and Sepsis Investigators (PALISI) Network. Effect of mechanical ventilator weaning protocols on respiratory outcomes in infants and children: a randomized controlled trial. JAMA 2002;288(20):2561-2568.

8. Willson DF, Thomas NJ, Markovitz BP, Bauman LA, DiCarlo JV, Pon S, et al; Pediatric Acute Lung Injury and Sepsis Investigators (PALISI) Network. Effect of exogenous surfactant (calfactant) in pediatric acute lung injury: a randomized controlled trial. JAMA 2005;293(4):470-476.

9. Randolph AG, Forbes PW, Gedeit RG, Arnold JH, Wetzel RC, Luckett PM, et al; Pediatric Acute Lung Injury and Sepsis Investigators (PALISI) Network. Cumulative fluid intake minus output is not associated with ventilator weaning duration or extubation outcomes in children. Pediatr Crit Care Med 2005;6(6):642-647.

10. Burr JS, Jenkins TL, Harrison R, Meert K, Anand KJ, Berger JT, et al; Eunice Kennedy Shriver National Institute of Child Health and Human Development (NICHD) Collaborative Pediatric Critical Care Research Network (CPCCRN). CPCCRN Critical Pertussis Study: Collaborative research in pediatric critical care medicine. Pediatr Crit Care Med 2010. [epub ahead of print].

11. Meert KL, Eggly S, Dean JM, Pollack M, Zimmerman J, Anand KJ, et al. Ethical and logistical considerations of multicenter parental bereavement research. J Palliat Med 2008;11(3):444-450.

12. Meert KL, Eggly S, Pollack M, Anand KJ, Zimmerman J, Carcillo J, et al; National Institute of Child Health and Human Development Collaborative Pediatric Critical Care Research Network. Parents' perspectives regarding a physician-parent conference after their child's death in the pediatric intensive care unit. J Pediatr 2007; 151(1):50-55.

13. Willson DF, Dean JM, Newth C, Pollack M, Anand KJ, Meert K, et al; Collaborative Pediatric Critical Care Research Network (CPCCRN). Pediatr Crit Care Med 2006;7(4):301-307.

14. Yung M, Slater A, Festa M, Williams G, Erickson S, Pettila V, et al; Australia and New Zealand Intensive Care Influenza Investigators and the Paediatric Study Group and the Clinical Trials Group of the Australia New Zealand Intensive Care Society. Pandemic H1N1 in children requiring intensive care in Australia and New Zealand during winter 2009. Pediatrics 2011;127(1):e156-e163.

15. The ANZIC Influenza Investigators. Critical care services and 2009 H1N1 influenza in Australia and New Zealand. N Engl J Med 2009; 361(20):1925-1934.

16. Erickson S, Schibler A, Numa A, Nuthall G, Yung M, Pascoe E, Wilkins B; Paediatric Study Group, Australian and New Zealand Intensive Care Society. Acute lung injury in pediatric intensive care in Australia and New Zealand: A prospective, multicenter, observational study. Pediatr Crit Care Med 2007;8(4):317-323.

17. Zimmerman JJ, Akhtar SR, Caldwell E, Rubenfeld GD. Incidence and outcomes of pediatric acute lung injury. Pediatrics 2009;124(1): 87-95.

18. Cheifetz IM. Management of acute lung injury: sharing data between adults and children. Respir Care 2011;56(9):1258-1268; discussion 1268-1272.

19. The Acute Respiratory Distress Syndrome Network. Ventilation with lower tidal volumes as compared with traditional tidal volumes for acute lung injury and the acute respiratory distress syndrome. N Engl J Med 2000;342(18):1301-1308.

20. Brower RG, Lanken PN, MacIntyre N, Matthay MA, Morris A, Ancukiewicz M, Schoenfeld D, Thompson BT on behalf of the National Heart, Lung, and Blood Institute ARDS Clinical Trials Network. Higher versus lower positive end-expiratory pressures in patients with the acute respiratory distress syndrome. N Engl J Med 2004;351(4):327-336. 
21. Meade MO, Cook DJ, Guyatt GH, Slutsky AS, Arabi YM, Cooper DJ, et al. Ventilation strategy using low tidal volumes, recruitment maneuvers, and high positive end-expiratory pressure for acute lung injury and acute respiratory distress syndrome. JAMA 2008;299(6): 637-645.

22. Mercat A Richard JC, Vielle B, Jaber S, Osman D, Diehl JL, et al. on behalf of the Expiratory Pressure (Express) Study Group. Positive end-expiratory pressure setting in adults with acute lung injury and acute respiratory distress syndrome: a randomized controlled trial. JAMA 2008;299(6):646-655.

23. DiBlasi RM. Neonatal noninvasive ventilation techniques: do we really need to intubate? Respir Care 2011;56(9):1273-1294; discussion 1295-1297.

24. Brown MK, DiBlasi RM. Mechanical ventilation of the premature neonate. Respir Care 2011;56(9):1298-1311; discussion 1311-1313.

25. Oishi P, Datar SA, Fineman JR. Advances in the management of pulmonary hypertension. Respir Care 2011;56(9):1314-1339; discussion 1339-1340.

26. Marecki JC, Cool CD, Parr JE, Beckey VE, Luciw PA, Tarantal AF, et al. HIV-1 Nef is associated with complex pulmonary vascular lesions in SHIV-nef-infected macaques. Am J Respir Crit Care Med 2006;174(4):437-445.

27. Graham BB, Mentink-Kane MM, El-Haddad H, Purnell S, Zhang L, Zaiman A, et al. Schistosomiasis-induced experimental pulmonary hypertension: role of interleukin-13 signaling. Am J Pathol 177(3): 1549-1561.

28. Souza R, Humbert M, Sztrymf B, Jais X, Yaici A, Le Pavec J, et al. Pulmonary arterial hypertension associated with fenfluramine exposure: report of 109 cases. Eur Respir J 2008;31(2):343-348.

29. Gentile MA. Inhaled medical gases: more to breathe than oxygen. Respir Care 2011;56(9):1341-1357; discussion 1357-1359.

30. Ballard RA, Truog WE, Cnaan A, Martin RJ, Ballard PL, Merrill JD, et al. Inhaled nitric oxide in preterm infants undergoing mechanical ventilation. N Engl J Med 2006;355(4):343-353.

31. Kinsella JP, Cutter GR, Walsh WF, Gerstmann DR, Bose CL, Hart $\mathrm{C}$, et al. Early inhaled nitric oxide therapy in premature newborns with respiratory failure. N Engl J Med 2006;355(4):354-364.

32. Van Meurs KP, Wright LL, Ehrenkranz RA, Lemons JA, Ball MB, Poole WK, et al. Inhaled nitric oxide for premature infants with severe respiratory failure. N Engl J Med 2005;353(1):13-22.

33. Dellinger RP, Zimmerman JL, Taylor RW, Straube RC, Hauser DL, Criner GJ, et al. Effects of inhaled nitric oxide in patients with acute respiratory distress syndrome: results of a randomized, phase II trial. Crit Care Med 1998;26(3):15-23.

34. Taylor RW, Zimmerman JL, Dellinger RP, Straube RC, Criner GJ, Davies K Jr., et al. Low-dose inhaled nitric oxide in patients with acute lung injury: a randomized controlled trial. JAMA 2004;291(13): 1603-1609.

35. Gerlach H, Pappert D, Lewandowski K, Rossaint R, Falke KJ. Longterm inhalation with evaluated low doses of nitric oxide for selective improvement of oxygenation in patients with adult respiratory distress syndrome. Intensive Care Med 1993;19(8):443-449.

36. Michael JR, Barton RG, Saffle JR, Mone M, Markewitz BA, Hillier $\mathrm{K}$, et al. Inhaled nitric oxide versus conventional therapy: effect on oxygenation in ARDS. Am J Respir Crit Care Med 1998;157(5 Pt 1): $1372-1380$.

37. Gerlach H, Keh D, Semmerow A, Busch T, Lewandowski K, Pappert $\mathrm{DM}$, et al. Dose-response characteristics during long-term inhalation of nitric oxide in patients with severe acute respiratory distress syndrome: a prospective, randomized, controlled study. Am J Respir Crit Care Med 2003;167(7):1008-1015.

38. McIntyre RC, Moore FA, Moore EE, Piedalue F, Haenel JS, Fullerton DA. Inhaled nitric oxide variably improves oxygenation and pulmonary hypertension in patients with acute respiratory distress syndrome. J Trauma 1995;39(3):418-425.

39. Papazian L, Bregeon F, Gaillat F, Thirion X, Gainnier M, Gregoire $\mathrm{R}$, et al. Respective and combined effects of prone position and inhaled nitric oxide in patients with acute respiratory distress syndrome. Am J Respir Crit Care Med 1998;157(2):580-585.

40. Krafft PP, Fridich RD, Fitzgerald RD, Koc D, Stelzer H. Effectiveness of nitric oxide inhalation in septic ARDS. Chest 1996;109(2): 486-493.

41. Keidan I, Mishaly D, Berkenstadt H, Perel A. Combining low inspired oxygen and carbon dioxide during mechanical ventilation for the Norwood procedure. Paediatr Anaesth 2003;13(1):58-62.

42. Graham EM, Bradley SM, Atz AM. Preoperative management of hypoplastic left heart syndrome. Expert Opin Pharmacother 2005; 6(5):687-693.

43. Tabbutt S, Ramamoorthy C, Montenegro LM, Durning SM, Kurth $\mathrm{CD}$, Steven JM, et al. Impact of inspired gas mixtures on preoperative infants with hypoplastic left heart syndrome during controlled ventilation. Circulation 2001;104(12 Suppl 1):I159-I164.

44. Toiyama K, Hamaoka K, Oka T, Kobayashi N, Noritake K, Kato R, et al. Changes in cerebral oxygen saturation and blood flow during hypoxic gas ventilation therapy in HLHS and CoA/IAA complex with markedly increased pulmonary blood flow. Circ J 2010;74(10): 2125-2131.

45. Myers TR, Chatburn RL. Accuracy of oxygen analyzers at subatmospheric concentrations used in treatment of hypoplastic left heart syndrome. Respir Care 2002;47(10):1168-1172.

46. Dolinay T, Szilasi M, Liu M, Choi AM. Inhaled carbon monoxide confers anti-inflammatory effects against ventilator-induced lung injury. Am J Respir Crit Care Med 2004;170:613-620.

47. Nemzek JA, Fry C, Abatan O. Low-dose carbon monoxide treatment attenuates early pulmonary neutrophil recruitment after acid aspiration. Am J Physiol Lung Cell Mol Physiol 2008;294(4):L644-653.

48. Otterbein LE, Mantell LL, Choi AM. Carbon monoxide provides protection against hyperoxic lung injury. Am J Physiol 1999;276: L688-L694.

49. Kohmoto J, Nakao A, Stolz DB, Kaizu T, Tsung A, Ikeda A, et al. Carbon monoxide protects rat lung transplants from ischemia-reperfusion injury via a mechanism involving p38 MAPK pathway. Am J Transplant 2007;7(10):2279-2290.

50. Fujita T, Toda K, Karimova A, Yan SF, Naka Y, Yet SF, et al. Paradoxical rescue from ischemic lung injury by inhaled carbon monoxide driven by depression of fibrinolysis. Nat Med 2001;7(5): 598-604.

51. Wiswell TE. Resuscitation in the delivery room: lung protection from the first breath. Respir Care 2011;56(9):1360-1367; discussion 1367-1368.

52. Kattwinkel, J and the AHA/AAP Neonatal Resuscitation Program Steering Committee, editors. Textbook of neonatal resuscitation, 6th edition. Elk Grove Village, IL: American Academy of Pediatrics; 2011.

53. Willson DF, Notter RH. The future of exogenous surfactant therapy. Respir Care 2011;56(9):1369-1386; discussion 1386-1388.

54. Myers TR, Tomasio L. Asthma: 2015 and beyond. Respir Care 2011; 56(9):1389-1407; discussion 1407-1410.

55. Spycher BD, Silverman M, Kuehni CE. Phenotypes of childhood asthma: are they real? Clin Exp Allergy 2010;40(8):1130-1141.

56. Valerio MA, Andreski PM, Schoeni RF, McGonagle KA. Examining the association between childhood asthma and parent and grandparent asthma status: implications for practice Clin Pediatr (Phila) 2010; 49(6):535-541.

57. Berg AO, Baird MA, Botkin JR Driscoll DA, Fishman PA, Guarino $\mathrm{PD}$ et al. National Institutes of Health State-of-the-Science Confer- 


\section{Neonatal and Pediatric Respiratory Care: What Does the Future Hold?}

ence Statement: family history and improving health. Ann Intern Med 2009;151(12):872-877.

58. Yang KD. Concensus. Limitations and Perspectives on Pediatric Asthma Treatment. Pediatr Neonatol 2010;51(1):5-6.

59. Ober C, Hoffjan S. Asthma genetics 2006: the long and winding road to gene discovery. Genes Immun 2006;7(2):95-100.

60. Bisgaard $\mathrm{H}$, Bonnelykke K. Long-term studies of the natural history of asthma in childhood. J Allergy Clin Immunol 2010;126(2):18797.

61. Strachan DP. Family size, infection and atopy: the first decade of "hygiene hypothesis". Thorax 2000;55(Suppl I):2-10.

62. Strachan DP, Cook DG. Health effects of passive smoking. 5. Parental smoking and allergic sensitization in children. Thorax 1998; 53(2):117-123.

63. Andersson M, Bjerg A, Forsberg B, Lundback B, Ronmark E. The clinical expression of asthma in schoolchildren has changed between 1996-2006. Pediatr Allergy Immunol 2010;21(5):859-866.

64. Akinbami LJ, Moorman JE, Garbe PL, Sondik EJ. Status of childhood asthma in the United States, 1980-2007. Pediatrics 2009; 123(Suppl 3):S131-S145.

65. Rubin, BK. Pediatric aerosol therapy: new devices and new drugs. Respir Care 2011;56(9):1411-1421; discussion 1421-1423.

66. Walsh BK, Hood K, Merritt G. Pediatric airway maintenance and clearance in the acute care setting: how to stay out of trouble. Respir Care 2011;56(9):1424-1440; discussion 1440-1444.

67. Seymour CW, Cross BJ, Cooke CR, Gallop RL, Fuchs BD. Physiologic impact of closed system endotracheal suctioning in spontaneously breathing patients receiving mechanical ventilation. Respir Care 2009;54(3):367-374.

68. Copnell B. Commentary: Morrow B, Futter M, Argent A. Effect of endotracheal suction on lung dynamics in mechanically-ventilated paediatric patients. Nurs Crit Care 2008;13(1):54-55.
69. Dalton HJ. Extracorporeal life support: moving at the speed of light. Respir Care 2011;56(9):1445-1453; discussion 1454-1456.

70. Domico MD, Ridout DA, Bronicki R, Anas NG, Cleary JP, Cappon J, Goldman AP, Brown KL. The impact of mechanical ventilation time before initiation of extracorporeal life support on survival in pediatric respiratory failure: a review of the ELSO registry. Pediatr Crit Care Med 2011 [Epub ahead of print].

71. Peek GJ, Mugford M, Tiruvoipati R, Wilson A, Allen E, Thalanany $\mathrm{MM}$, et al. Efficacy and economic assessment of conventional ventilatory support versus extracorporeal membrane oxygenation for severe adult respiratory failure (CESAR): a multicentre randomised controlled trial. Lancet, 2009;374(9698):1351-1363. Erratum in Lancet 2009;37(9698):1330.

72. Turner DA, Cheifetz IM, Rehder KJ, Williford WL, Bonadonna D, Banuelos SJ, et al. Active rehabilitation and physical therapy during extracorporeal membrane oxygenation while awaiting lung transplantation - a practical approach. Crit Care Med 2011 (in press).

73. Turner DA, Williford WL, Peters MA, Thalman JJ, Shearer IR, Walczak RJ Jr, et al. Development of a collaborative program to provide extracorporeal membrane oxygenation for adults with refractory hypoxemia within the framework of a pandemic. Pediatr Crit Care Med. 2011;12(4):426-430.

74. Branson RD. Disaster planning for pediatrics. Respir Care 2011; 56(9):1457-1463; discussion 1463-1465.

75. Gausche-Hill M. Pediatric disaster preparedness: are we really prepared? J Trauma 2009;67(2 Suppl):S73-S76.

76. Gausche-Hill M, Schmitz C, Lewis RJ. Pediatric preparedness of US emergency departments: a 2003 survey. Pediatrics 2007;120(6):12291237.

77. Gausche-Hill M, Wiebe RA. Guidelines for preparedness of emergency departments that care for children: a call to action. Pediatrics 2001;107(4):773-774. 